\title{
LA PROBLEMÁTICA ÉTICA EN EL PENSAMIENTO DE HUSSERL
}

\author{
JULIA VALENTINA IRIBARNE
}

Asociación Argentina de InVEstigaciones Éticas

Abordar el tema de la filosofía práctica en Husserl exige proyectar una mirada abarcadora a los diversos ámbitos de cuestiones hacia los que el filósofo orientó su pensamiento. Una afirmación se presenta en primer lugar como incuestionable y es que en ningún momento el desarrollo de la problemática abandona el ámbito de exégesis de la constitución del objeto, incluso vale decir que nunca se sustrae al marco de la filosofía trascendental. En consecuencia, en Husserl la filosofla práctica tendrá este carácter.

En general, el objeto ha valido en fenomenología como hilo conductor hacia el funcionamiento noético-noemático del que resultaba. En el tema de que nos ocupamos es posible extender este punto de vista a la posición de los valores y descubrir las funciones que la hacen posible. Ese fue el tema de Husserl en sus Lecciones de ética. No es ése el que nosotros nos proponemos abordar en esta oportunidad. Sin embargo, mantendremos nuestra consideración dirigida a la problemática de la constitución. Por esa vía se nos hace manifiesto que la cuestión ética, vale decir, la pregunta por el Bien, por el error y la verdad, en relación con una existencia auténtica aparecen en un alto estrato de constitución. Parecerán no plantearse problemas éticos en la medida en que reflexionemos sobre la constitución de los objetos ni cuando atendamos a la legitimación del Otro trascendental. Las cuestiones éticas se hacen manifiestas en cuanto abordamos el alto estrato de constitución de la persona en el mundo social.

Si en general las áreas abordadas por la filosofía práctica pueden diferenciarse según su alcance como microámbito, mesoámbito y macroámbito, es decir, ámbito de la persona singular, de las naciones o pueblos y planetario respectivamente, es nuestra intención mostrar que en el caso de Husserl la cuestión del macroámbito tiene un valor predominante, que no es separable del microámbito, en tanto que el mesoámbito tiene un sentido de mediación, destinada ésta a ser reabsorbida por el nivel superior. Esto equivale a afirmar que la mayor preocupación de Husserl como pensador ético se vincula a la idea 
-en sentido kantiano- de una convivencia universal armónica, que abarque en sí a pueblos y naciones y a las personas que los configuran.

"El mundo" tiene, desde este punto de vista, un valor ético precisamente porque exige ser pensado unitariamente. ${ }^{1}$ Un mundo en colapso, desorganizado, del que esté ausente la tendencia a una configuración de convivencia universalmente articulada, no sería ya más un mundo. Dicho en sentido inverso, parte del sentido unitario de "mundo"-procede de una teleología unificante inmanente al sujeto constituyente.

El planteamiento que abordamos no nos es ofrecido por Husserl en ninguna exposición sistemática. Es nuestra intención, en consecuencia, unir índices precisos pero dispersos.

\section{La cuestión del sumo Bien}

La circunscripción de una toma de posición ética comienza por la definición de lo que se entiende por Bien, mejor aún, por Sumo Bien. El Sumo Bien es para Husserl una idea que entraña en sí misma rasgos de universalidad y, al mismo tiempo, del más concreto contenido: lo que él designa como “Todo de las mónadas. Se trata de la omniabarcadora problemática que también puede denominarse con el titulo de 'teleologia universal'. Dicho de otro modo, son estos los problemas de la totalidad, de la posibilidad trascendental de una intersubjetividad existente, abierta, infinita, inclusida alls la pasibilidad de la verdadeva 'preservación de si misno' (Erhaltung) por parte de cada subjetividad individual y social en interconexión infinita. Los problemas de la armonia universal pero tambión los de la auténtica humanidad alcanzan entonces, como problemas fenomenologicos, su sentido absoluto, referido a la subjetividad trascendental. Asi, la más alta conclusion para la problemática de la filosofta fenomenologica es la pregunta por el 'principio' de la teleologia, concretamente inclusida en sus estructuras universales". 2 De acuerdo con lo aquí expresado, "teleología" es otra denominación para el "todo de las monadas".

\section{Manifestación del telos a la analítica fenomenologica}

Husserl toma el concepto de teleología en su sentido corriente de acción orientada hacia un fin. Ella se manifiesta en todos los estratos que emergen a la luz de la reducción fenomenológica: de manera "admirable" en el mundo de la vida gracias a sistemas de función que se realizan en relación con una meta; la percepción, por su parte, se ofrece como un proceso teleológico, tal como se exhibe en las síntesis pasivas; el verdadero ser del mundo es un telos unificado

1 E. Husserl, Zur Pbennomenrologic der Intersubjetipitit, Parte III, M. Nijhoff, La Haya, 1974. p. 214.

2 Carta a E. Pearl Welch, escrita entre el 17 y el 21 de junio de 1933 , Op. cit., pp. LVIII-LIX. 
en lo infinito de un proceso de una realización siempre más perfecta, que continuia Libremente. ${ }^{3}$ En general, podemos afirmar que la mención de Husserl de la teleología intrínseca a la intencionalidad, se vincula a la aspiración originaria de conocimiento que gobierna en el mentar dóxico de la más baja esfera de representación y determina la pluralidad de los diversos grados de la vida intencional. ${ }^{4}$ Baste esta somera mención de la presencia universal del telos en los estratos más bajos de constitución trascendental, para articular con ellos el estrato más alto, el que estamos considerando, en particular a partir de un texto del 5 de noviembre de $1931.5^{5}$ Allí se nos dice que el proceso de desarrollo de la subjetividad trascendental, o sea, de la intersubjetividad trascendental, trae en sí una voluntad respecto del verdadero ser, que se hace patente primero en los sujetos singulares, en cuya voluntad, en su forma patente, despierta un horizonte de voluntad latente como horizonte de la auténtica humanidad: la idea del ser subjetivo singular perfecto dentro de una perfecta comunidad total infinita que no es pensable como una forma realizada. Se trata de la idea de un existente, el más perfecto de todos, en infinito progreso que supera todas las contradicciones del existente. Hay, para Husserl, un despertar de la teleología inmanente en la intersubjetividad trascendental, teleología que se muestra como forma de todas las formas y exhibe su tracción en la historicidad de la intersubjetividad. Husserl expresa el devenir de este proceso hacia la verdad a través de la voluntad, con conceptos, si no crípticos, por lo menos inorgánicos, de los que nos atrevemos a dar una interpretación. El sentido de "verdad" que vive en la intersubjetividad está en la voluntad universal trascendental entrañada en ella y deviene en grados de vigilia de "voluntad despierta" - 'en la forma de la 'verdadera' tradicionalidad, como tradicionalidad de la verdad que estai solamente en la voluntad. La poluntad en el perdadero yo, el que se ba hecho cargo de su ser intersubjetivo, de alcanzar una verdadera comunidad, es voluntad 'en sentido propio'". 6 En este texto tradicionalidad resulta otra denominación para la intersubjetividad como trama que sostiene a cada yo singular con el bagaje cultural total, a partir del cual cada ego "despierto" tenderá hacia la conjunción perfecta de todos los alteri pensables.

\section{Interrelación de microámbito, mesodmbito y macroímbito.}

Lo afirmado hasta aquí nos permite avanzar en la comprensión de la posición del macroámbito en la filosofía práctica de Husserl. También comienza a insinuarse su relación con el mesoámbito y el macroámbito. Ésta procede de

3 E. Husserl, Este Philosopbic I, M. Nijhoff, La Haya, 1956. pp. 274 y s. p. 143.

4 G. Hoyos Vásquez, Intentionalitait als Verantwortung, M. Nijhoff, La Haya, 1976,

5 Zur Phïnomenologie der Intersubjetivität. Parte III, pp. 378 y ss.

6 Op. cit., p. 379. 
que la configuración del Todo de las mónadas subsume en unidad concordante con todos los pueblos y naciones; éstos últimos, denominados por el filósofo personalidades de orden superior, son tales en la medida en que están sostenidos por la común voluntad ${ }^{7}$ de las personas. A esta común voluntad, a esta posición volitiva como intencionalidad "volente" de los vivientes en sociedad, es a la que se nos remite cuando en nuestro retropreguntar partimos del objetofamilia, objeto-Iglesia, objeto-Estado como hilo conductor. Cualquiera que sea la configuración respectiva, debe su actualidad y su vigencia a la viviente voluntad que la sostiene y, viceversa, su ausencia la descubre como una cáscara vacía.

Esta voluntad común de un grupo, sin embargo, si no ostenta más títulos que los hasta aquí elucidados, no garantiza ni convalida el sentido de su volición. Es posible concebir una comunidad volitiva aplicada a la destrucción de otra comunidad, por no recurrir a ejemplos de la historia pasada y presente. Lo que equivale a decir que la comunidad volitiva no es necesariamente ética. ¿Qué es lo que le otorga este carácter?

En sus reflexiones sobre el amor entendido como un ejemplo de comunidad volitiva, Husserl privilegia el amor cristico. ${ }^{8}$ Cristo es el paradigma de la persona que da y suscita amor y esto sin restricciones. El amor como sentimiento tiene valor en la experiencia personal, sin embargo, no es sobre este aspecto sobre el que se apoya el filósofo. Por un lado, el amor exhibe la coincidencia volitiva, por el otro, y esto es lo que a Husserl le parece esencial en la figura crística, es una relación, concretamente universalizada en Cristo, que expresa la asunción del amor al prójimo como a sí mismo, en una paridad de términos insoslayable. Tal universalidad irrestricta se expresa en el amor al enemigo. El Cristo ama al enemigo no porque sea tal sino porque ama en él, como en todos, el alma germinal en la que apunta al ideal de cada uno, como a su propia vida en autenticidad.

Consecuentemente, podemos afirmar que este rasgo del amor universal ha de formar parte esencial de la comunidad volitiva ética, así como de cada persona moral. Intentaremos a continuación delinear los rasgos de la persona moral sabiendo que, si bien no hallamos en Husserl una doctrina elaborada a ese respecto, sí encontramos en su pensamiento sus aspectos configurativos.

El primer rasgo concierne a la estructura temporal del ser humano que, como dirá después de Husserl la filosofía de la existencia, "tiene que hacer su ser en lugar de serlo simplemente”. No son pocas las referencias husserlianas en este sentido. El sujeto, que cuenta entre sus actos constitutivos sus valoraciones, sus voliciones así como sus decisiones, elige sus motivos ${ }^{9}$ No se afirma

7 Zur Phanomenologie..., Parte II, pp. 179-180.

8 Op. cit., p. 174.

9 Zur Phänomenologie..., Parte I, p. 93 y siguientes. 
que la persona sea un sujeto totalmente libre en la medida en que conoce y expone la génesis de sus habitualidades, que funcionan en él como una carga determinante. Sin embargo, en la interrelación con el Otro, sea personal o por mediación de los objetos culturales, no está determinado sino motivado. Determinación y motivación operan de modo diferente: Estoy en mi casa, dispuesto a pasar una tarde en la que voy a dar fin a una tarea. Cae un rayo, la casa comienza a incendiarse, la abandono precipitadamente. Así funciona la determinación. En las mismas circunstancias y con el mismo proyecto, llaman a mi puerta, abro y recibo el telegrama enviado por un amigo que está muy enfermo y me reclama. Recojo lo necesario y parto. Así funciona la motivación. El Otro me ofrece un motivo que yo reasumo o no. Husserl sabe que nuestro margen de posibilidades "reales" es estrecho pero también sabe que lo hay. En esa medida, el hombre se le aparece como libre y puede llegar a ser persona moral. Este advenimiento se vincula a lo que aludimos como alma germinal, a la orientación hacia un telos activo en cada hombre como un horizonte potencial abierto hacia una mayor perfección, hacia una existencia siempre más auténtica.

Esta afirmación vuelve a llevarnos a la cuestión del fundamento valorativo que decide qué es lo que aproxima o aleja de una existencia humana auténtica, tal como decide el valor de una comunidad volitiva. Llegados a este punto y sin otra referencia que la del macroámbito como ámbito ideal de realización de la convivencia universal armónica, parece necesario afirmar que, consecuente con ese telos, cada hombre en su existencia personal debe inscribirse en tal dirección, que cada uno es responsable por su parte de esa realización solidaria. Pero todavía cabe preguntarse por qué la persona debe acatar la norma según la cual su valor sea igual al de todos los demás hombres y de acuerdo con la cual no puede privilegiar su posición sin traicionarlos y traicionarse.

\section{La experiencia intersubjetiva como criterio de validez moral.}

Desde nuestra comprensión del pensamiento de Husserl, nos vemos precisados a afirmar que la experiencia que funciona como clave de bóveda para este reconocimiento fundamental es la de la referencia recíproca de constitución entre las personas.

La relación yo-tú, infinitamente universalizable, expresa la dependencia recíproca en el reconocimiento personal. El Otro es el primer bombre, no yo. ${ }^{10}$ Esto quiere decir que a pasteriori, de haberlo constituido como hombre, sujeto de un mundo social que no es diverso del mío, mundo en el cual aparezco a él a mi vez como hombre, es como yo alcanzo a comprenderme a mí mismo como tal. Es la posibilidad del Otro de constituirme como hombre la que 
me permite verme a mí mismo desde mí mismo como desde un tercero. Mi propio carácter de ser hombre pasa por mi previo reconocimiento en el Otro de ese carácter y con ello queda firmemente asentada nuestra equi-valencia radical.

La fenomenología de la intersubjetividad, llevada a cabo exhaustivamente por el filósofo, descubre en todos los estratos de constitución develados por la reducción fenomenológica la llamada estructura de repetición. Ella opera en cada caso de modo tal que cuando, desde mi esfera primordial, pongo al Otro-allá como alter ego, es decir, como un yo que gobierna un cuerpo-propio, punto cero de un mundo al que centra desde su aqut, aparezco y me aparezco a mi vez como si fuera el Otro para él. Este reconocimiento apareante es el fundamento del modo categórico con que debo afirmar que lo que tiene valor fundamental para mí puede, de jure, tenerlo también para él. Mis derechos fundan sus derechos. Esta estructura de repetición es, entonces, desde nuestro punto de vista, la que funda la asunción de mi equivalencia respecto de todos los seres humanos y el reconocimiento de un debido actuar en consecuencia.

\section{5. ¿Impera una universalidad "restringida" en el pensamiento de Husserl?}

Una última cuestión debe aún elucidarse: es la del alcance universal de la propuesta husserliana, habida cuenta de su referencia a la humanidad europea, cuando en sus Crisis ${ }^{11}$ aborda la temática de la que nos estamos ocupando.

Se trata de alcanzar la justificación de la extensión del sentido bumanidad europea, con que él opera, al de bumanidad actual.

Husserl hace referencia a la humanidad europea, pues está convencido de que Europa es la heredera de un desarrollo histórico del espíritu cuyo giro peculiar comenzará en la antigua Grecia: “Una teleologla notable que solo pertenece por naturaleza a Europa, y que precisamente es intimamente solidaria de la aparición o de la irrupción de la filosofia y sus ramificaciones, las ciencias". ${ }^{12}$ Aclara que no toma la figura espiritual de Europa en un sentido geográfico. $\mathrm{Al}$ ámbito mencionado pertenecen los individuos singulares que actúan en él, "en muiltiples sociedades de diverso grado, en familias, razas, naciones, todas ligadas intimamente por el espiritu y... reunidas en una forma espiritual". ${ }^{13}$ Es en esta afirmación donde comenzamos por apoyar nuestra extensión del planteamiento de la humanidad actual. Por otra parte, tomamos en consideración que en nuestros días el progreso de los medios ha comunicado, en sentido concreto, a prácticamente todas las zonas de la tierra. El acceso al ejercicio del

11 Nos referimos a Die Krisis der curopdischen Wioconschaften und die transzendentale Phdinomenologic y a Dic Krisis des europdischen Menschentums und die Philosophic, M. Nijhoff, La Haya, 1969.

12 Die Krisis des europaischen Menschentums..., p. 318.

13 Op. cit., p. 319. 
pensar libre y crítico, a la ciencia y a sus aplicaciones técnicas es, de derecho, posible en toda la superficie del planeta. Del mismo modo, las consecuencias del grado de desarrollo de ciencia y técnica someten a la tierra entera a una misma amenaza. En vista de este estado de cosas mundano, resulta posible extender las reflexiones del filósofo a la problemática de la humanidad que ha heredado, para su bien y para su mal, los logros de la trayectoria del espíritu.

No nos detenemos en la descripción pormenorizada de la situación que nos permite afirmar que el mundo se ha unificado bajo el signo de la ciencia y de la técnica, en un sentido que, como ya habría de denunciar Husserl, traiciona el espíritu del cual nació. ¿En qué se expresa tal traición, tal olvido? Para responder a esta pregunta recorremos el mismo camino que el filósofo.

Lo peculiar de la humanidad europea es que "entraña una entelequia que le es innata, que domina todos los cambios que afectan a Europa y le confiere un sentido, el de un desarrollo orientado bacia un polo eterno". ${ }^{14}$ Su comienzo se confunde con el de la filosofía y las ciencias que de ella se desprendieron en el alba de la cultura griega. En ese momento el sentido con que nace la filosofía es el de "ciencia universal, ciencia del todo del mundo, de la totalidad de los existentes". ${ }^{15}$ El telos espiritual o la entelequia es una idea infinita hacia la que se orienta el desarrollo histórico del espíritu, en el ámbito práctico es un fin propuesto por la voluntad y se presenta como una nueva forma del desarrollo que se halla bajo el control de normas, de ideas normativas. El permanente "estar dirigido hacia" una norma, propio de la vida intencional de las personas singulares, pasa a las naciones y finalmente al organismo que las abarque (Europa, textualmente, en sentido lato). La humanidad en su conjunto es progresivamente reformada por esta particularidad admirable y nueva de entrañar una infinitud intencional, el hombre deviene un hombre nuevo. El hombre crea ideales en dirección a objetivos siempre nuevos, de grado siempre más alto, que constituyen una tarea infinita. No se trata, en este contexto, de una especulación abstracta, sino de la poderosa transformación cultural que irradia la filosofía: busca la verdad en sí, incondicionada, y la busca desde una actitud crítica, con intención de someter el conjunto de la experiencia a normas ideales: "la verdad incondicionada... provoca inmediatamente una transformación abarcadora de toda la praxis del existente bumano, vale decir, de toda la vida cultural'. ${ }^{16}$ A partir de esta orientación hacia ideas de que todos los hombres participan, se produce un peculiar actuar comunitario: el del trabajar con el Otro y para el Otro, el del ejercicio de una crítica constructiva respecto del Otro, en el que se cultiva la validez pura e incondicionada de la verdad como un bien común. A partir de aquí puede surgir una sobrenacionalidad de un tipo 
completamente nuevo..., "existen ideales infinitos para la sintesis cada vez más vasta de las naciones; por el becho de que cada una de ellas abarca su propia tarea ideal seguin el espiritu de infinitud, ella bace donación de lo mejor que tiene a las naciones asociadas... La filosofia tiene que cjercitar su función permanentemente en una bumanidad europea, como arcóntica respecto de toda la bumanidad". ${ }^{17}$

Husserl está convencido de que la crisis europea - ya podemos decir mundial - arraiga en un racionalismo equivocado. El racionalismo de la ciencia ha abandonado su pretensión universal y su solidaridad con la búsqueda de una verdad incondicional y la persecusión de ideales como tarea infinita. "Ast, la racionalidad unilateral puede convertirse en un mal", ${ }^{18}$ en tanto la auténtica racionalidad está llamada a "guiar el desarrollo de la bumanidad por la via de su madurez". ${ }^{19}$ Precisamente esta carencia de una auténtica racionalidad en todos los planos es la fuente de la insoportable confusión del hombre acerca de su propia existencia y de su tarea infinita.

En síntesis: la razón en su auténtico y originario sentido es la “autocomprensión universal y efectivamente radical del espiritu, en forma de ciencia universalmente responsable". ${ }^{20}$ En vista de lo hasta aquí expuesto, la crisis sólo tiene dos salidas posibles: o Europa, la humanidad, "cae en la alienación respecto de su propio sentido de vida... o renace a partir del espiritu de la filosofia..."21

Este tema encuentra, circunstancialmente, objeciones. Las más frecuentes son dos. En primer lugar, se ha dicho que Husserl, instalado en su fuerte sentido de pertenencia a la humanidad europea, ha sido ciego para la comprensión del valor de otras formas espirituales. En este sentido habría practicado una suerte de "provincianismo", de "solipsismo comunitario". En segundo lugar, se ha dicho que su pretensión unificadora, pasa por encima de las identidades diferenciadas, como consecuencia de lo cual su pensamiento no podría albergar un provechoso pluralismo.

En cuanto a la primera objeción, concerniente al "provincianismo" del filósofo, su afirmación de la búsqueda de universalidad, de verdad incondicionada como ejercicio de coherencia, muestra que tal toma de posición elude toda parcialidad. El texto que tomamos en consideración, escrito en tiempos del ascenso al poder del nacional-socialismo, por un filósofo segregado por su raza, a quien se le había prohibido expresar públicamente sus ideas o editar sus libros, no hace sino señalar el camino de superación de toda parcialidad, de todo prejuicio sectorial. Quiere despertar en Europa el sentido de comunidad universal, el destino universalizante, que dos mil quinientos años antes

\footnotetext{
17 op. cit., p. 336.

18 Op. ait., p. 338.

19 Op. cit., p. 337.

20 Op. cit., p. 345.

21 Op. cit., p. 347-48.
} 
se había puesto en marcha. Insistir en el hecho de que su punto de partida es la humanidad europea y que a ella se dirige, y no reconocer que esto es así, porque ella asumió de hecho la posibilidad humana de esforzarse hacia un ideal de vigencia de unidad universal, como reino del espíritu, es tergiversar el sentido total de su pensamiento.

Pero tendencia hacia la unidad universal es sinónimo de tendencia hacia la ausencia de contradicciones y no equivale a la repetición de una identidad hacia el infinito. Por eso como respuesta a la segunda objeción, y fundándonos en sus reflexiones sobre la intersubjetividad, podemos afirmar que la filosofia de Husserl, considerada en su conjunto, es la superación de todo prejuicio que favorezca un modo de ser, así sea éste europeo, si esto se entiende como una voluntad "homogeneizante". La experiencia básica señalada, del entretejimiento originario de ego y alter en todos los estratos, hace que el individuo - sea hombre o comunidad singular - logre captar su propia identidad por su referencia a lo que es Otro para él, siendo ambos, en todos los casos, el nosotros primario. Precisamente, el sentido ideal del todo de las monadas es el de la posibilidad de toda individualidad de trascender hacia el Otro, y como dice el filósofo, darle lo mejor suyo. En un texto de 1921/22, destinado a formar parte de una obra sistemática para la que Husserl trabajó largamente, encontramos unas líneas que ilustran este punto de vista: el filósofo, que reflexiona sobre la comunidad cultural europea, se pregunta cómo concebir la comunidad con el bombre patagónico. Y responde: " 10 , como aleman, tengo sollo un borizonte de personalidades de pueblos o de comunidades de pueblos, y cada pueblo como unidad, puede baber construido en $s i$ mismo representaciones, en las cuales él está representado para $s$ mismo (autoconciencia equivalente a la conciencia yoica de un pueblo) y otras [representaciones] en las que son representables, son experimentables, los otros pueblos en forma del alter (otro pueblo)". ${ }^{22}$ Tal como hemos visto, el alter es para el filósofo alter ego y, por eso, en todos los casos configura con él el nosotros. El espíritu se desarrolla en la historia: el que es alter para un momento de desarrollo del espíritu es, por ser tal, apto para inscribirse en la búsqueda infinita de la verdad incondicionada que aproxime a la unidad. Desde el momento en que el nosotros se configura esencialmente con un entretejimiento de reciprocas alteridades, el pluralismo forma parte de su misma esencia. Nada más nos hace falta para asentar el alcance universal de la propuesta del fiósofo.

\section{A modo de conclusión}

Lo expuesto hasta aquí nos lleva a afirmar que, si fuera de los desarrollos orgánicos concernientes a la constitución de los valores, no encontramos en los textos de Husserl publicados hasta el presente una explícita arquitectónica 
de la razón práctica, tenemos en cambio los elementos que permiten delinear su pensamiento ético y la posición de su ética en la totalidad sistemática de su filosofía. Con la cuestión ética culmina el sistema y culmina a la manera husserliana, porque en los estratos más altos de constitución aparecen los temas ético-religiosos y éstos, como problemas, permanecen abiertos. La teleología no tiene vigencia determinante. Tal como se enuncia este planteamiento, es posible concluir que la intención última del pensamiento husserliano es ética. A este respecto es ineludible volver a su reiterada afirmación acerca de la función arcónticas de la filosofie, tal como ella teleológicamente ha devenido fenomenología. En un texto al que se hace referencia como su "testamento filosófico" nos dice: “En la temporatizacion 'bumana', como desarrollo de mónadas racionales de orden superior (vale decir como Todo de las mónadas bumanas), desarrollo como las mónadas 'arcónticas' de quienes son soportes singulares de la razion y 'conductores' - Los hombres de ciencia, los filbsofos, la comunidad fenomenologica". ${ }^{23}$ En los textos de ambas Crisis este tema es reiteradamente señalado. A quienes frecuentamos la fenomenología nos resulta comprometedora la afirmación concerniente a que la actitud fenomenológica total es llamada a provocar una completa transformación personal, que cobija el significado de una máxima transformación existencial. ${ }^{24}$ Nos vemos precisados, siguiendo al filósofo, a preguntarnos por qué no es lo mismo ser zapatero que fenomenólogo y esta pregunta, lejos de llevarnos hacia una vana gloria, por el contrario, hace que nos asomemos al ámbito inagotable de la responsabilidad: es que, desde el punto de vista de Husserl, precisamente es por la vía de la fenomenología como se abre el develamiento radical del sentido del ser, en el que apunta una coexistencia universal concordante que es nuestra tarea señalar. Este llamado a vivir despierto es, por lo tanto, un llamado a la responsabilidad y es ineludible aceptar que la tarea propuesta es una tarea con sentido, con el más amplio y más alto sentido concebible. La fenomenología es entonces la filosofía que va mostrando los pasos por los que se ha avanzado hasta este momento hacia la totalización abarcadora de pluralidades, como una posibilidad, la más humana, regida por un telos cuyo “llamado" puede, no obstante, malograrse. Los hombres podemos llevar al colapso a la humanidad; por eso es arcóntica la función de la filosofía como vía hacia el despertar de la autorresponsabilidad.

24 Dic Krisis der ezropëischers Wrosenschafters..., p. 140. 